\title{
Cancer in pregnancy
}

\author{
Gurpreet Singh
}

Received: 30 October 2009 / Accepted: 16 November 2009

(C) Association of Surgeons of India 2009

\begin{abstract}
Cancer during pregnancy is a rare situation which demands a multidisciplinary care involving the surgical oncologist, the medical oncologist, the obstetrician and a host of other care givers. The care of the patient is planned in a way to optimize the health care delivery to the mother without compromising on the care of the fetus. When this is not possible priorities need to be established and harsh decisions taken on an individual basis. The treatment of the cancer does not change from the standard outside the limits described above and the objective should be to stay as close to the standard of care treatment as possible.
\end{abstract}

Keywords Pregnancy $\cdot$ Cancer

G. Singh

Department of Surgery,

Postgraduate Institute of Medical Education and Research

(PGIMER), Chandigarh, India

G. Singh $(\bowtie)$

E-mail: gsinghpgi@sify.com

\section{Introduction}

The occurrence of cancer in pregnant women is not common. This is because the peak incidence of most malignancies does not coincide with the peak reproductive years of the female. This scenario may change because of the increasing trend for women to delay childbearing in the western world. However, the situation in our country is reassuring as it is very rare for a woman not to have a child by age 30 .

The co-occurrence of cancer and pregnancy is reported with an average frequency of one in 1,000 births. The most common malignancies associated with pregnancy include cervical cancer, breast cancer, melanoma, lymphomas and leukaemia. Among a total of 1,134 pregnant patients with cancer, more than $50 \%$ had breast or cervical cancer. Another $25 \%$ had lymphoma and leukaemia, while the remaining had melanoma, thyroid cancer and other tumours [1].

Cancer diagnosed during pregnancy is a dramatic event where the creation of a new life (the unborn child) will clash with the potential of death in the mother. As such the impact and stress of the illness will be felt not only by the patient but by her family and physician also. This is further compounded by the perception that there has to be choice between the life of the mother and the life of the yet unborn child. Factors which may impact the course of treatment may be social, religious, personal, and moral rather than medical alone. As such the treatment of this delicate situation is best done in a multidisciplinary environment such as to balance optimal maternal treatment with foetal well being.

The following optimal standards should always be followed:

- To treat curable malignant disease of pregnant women

- To protect the foetus from harmful effects of cancer treatment

- To retain the mother's reproductive system intact for future gestations 
The personal experience of individual clinicians is necessarily limited. It is estimated that for an obstetrician delivering about 250 babies a year, to diagnose two or three patients with pregnancy-associated breast cancer would take at least 40 years of clinical practice [1]. Limited experience has resulted in a scarcity of information on the optimum management of pregnant women with cancer. Reports of single cases, or small series, highlight the difficult decisions needed and the potential conflict between the management of the tumour and the avoidance of harm to the foetus.

It is impossible to discuss the entire range of the individual cancers and their management when associated with pregnancy in this article. I will attempt to highlight certain common features of management which could be applied to all cancers.

\section{Physiological changes during pregnancy}

The normal changes occurring during early pregnancy are the influence of hormonal excess. These include breast swelling and/or tenderness, fatigue, nausea and vomiting. Smooth muscle relaxation can cause constipation and ureteric dilatation. Increased skin pigmentation and darkening of benign moles may occur. The amount of total body water and plasma volume increases considerably by about $50 \%$. This increase is associated with a reduction in haematocrit and in breathlessness. Changes in immune competence have the potential to change maternal response to infections and malignancies. These physiological changes in pregnancy can result in delayed disease diagnosis, because non-specific symptoms and even haematological and biochemical abnormalities can be attributed to normal features of pregnancy by the patient and by physicians. Suspicions of malignancy during pregnancy are low and a late presentation, often with advanced cancer, is common.

\section{Therapeutic abortion}

The first issue to be considered is whether pregnancy affects the behaviour of the cancer. Many unconvincing hypotheses have been put forward regarding the influence of cancer on the pregnancy. The high concentrations of oestrogens during pregnancy could adversely affect breast or endometrial cancer. The high concentration of growth factors could also potentially stimulate tumour growth. Immunological suppression during pregnancy could adversely affect cancer outcome. Increased tissue vascularity may increase the potential for metastases. Thus therapeutic abortion should be beneficial to the patient, but there is lack of evidence in this regard provided the patients receive the full antineoplastic treatment $[2,3]$.

Most women in India who develop pregnancy-associated cancer have completed their family by the time they develop cancer. The decision of therapeutic abortion is not such an ethical issue in this situation and is more acceptable to the patient, the family and the physician. The situation gets complicated if the woman has no prior children or desires to retain the current pregnancy. The decision for a therapeutic abortion in this setup is usually taken in some of the following settings:

- The pregnancy is an obstacle to the treatment of the cancer

- Advanced cancer with dismal prognosis

- Inadvertent exposure to high doses of radiation during first trimester

- Refusal of the parents to accept the risks of treatment for the foetus.

\section{Diagnostic workup}

The diagnostic workup of the pregnant woman with cancer should limit exposure to ionizing radiation and be restricted to procedures that do not endanger foetal health.

Ionizing radiation, whether diagnostic or therapeutic, can be harmful to the foetus. The risk and nature of adverse events are dependent on the radiation dose and the gestational age. During the first 2 weeks from conception irradiation of the undifferentiated embryo results in either death or normal development (all or none phenomenon) and doses as low as $100 \mathrm{mGy}$ can cause embryonic death, with a reported incidence of $50 \%$ with doses of $1 \mathrm{~Gy}$. During the period of organogenesis (weeks 3-12), embryonic death or developmental malformations are frequent with radiation doses of 1 Gy and may occur with doses as low as 50-250 mGy. The foetal dose of $100 \mathrm{mGy}(10 \mathrm{rad})$ during the first trimester is frequently cited as a threshold dose for the risk of teratogenesis. Exposure to $250 \mathrm{mGy}$ or higher doses of ionizing radiation during the second and third trimesters is associated with growth retardation, mental retardation, and premature birth. In cases of inadvertent exposure of pregnant women to radiation, termination of pregnancy is not recommended if the foetal dose does not exceed 100 mGy. Generally, doses $<100$ mGy (10 rads) are quite safe, as they are associated with a $<1 \%$ risk of malformation or carcinogenesis [4].

During pregnancy only absolutely necessary radiologic workup is justified. Chest X-ray seems safe with appropriate radioprotection. Two-view mammography, though associated with more intensive radiation exposure than plain chest X-rays, appears to be safe as well. Abdominal plain films, radionuclide isotope scans and computerised tomography (CT) scans should be avoided. For imaging of the brain, liver or bones in the context of clinical suspicion for metastases, magnetic resonance imaging (MRI) has been advocated. Gadolinium crosses the placenta and causes foetal abnormalities in rats, and thus is to be avoided [5].

Radionucleotides with short half lives (e.g. technetium99m) do not cause large foetal doses but iodine-131 crosses 
the placenta and is accumulated by the foetal thyroid and is associated with permanent hypothyroidism [4].

The radiation doses in sentinel node biopsy are sufficiently low for it to be done safely during pregnancy. The issue of intraoperative assessment to avoid a second anaesthesia for axillary dissection may obstruct the use of this procedure during pregnancy [6].

Endoscopy, lumbar puncture and bone marrow aspiration/biopsy are quite safe and should be done when clinically indicated, with appropriate caution to avoid excessive use of intravenous sedatives and opioids. Pulse oximetry monitoring should be used to avoid maternal and foetal hypoxia.

\section{Surgical treatment}

The surgical treatment of the cancer patient does not change because she is pregnant. The surgical procedure is tailored to the stage and need of the individual patient. All major surgical procedures can be carried out safely throughout pregnancy without additional risk to the mother.

A significant distinction between surgeries for intraabdominal or extra-abdominal malignancies during pregnancy is relevant. A laparotomy is more likely to interfere with pregnancy than an extra-abdominal surgical procedure. The enlarging uterus may make access to the involved organ difficult. An oncologically optimum resection may thus become technically more difficult. The uterus might need retraction, but care is essential to avoid impairment of placental flow or placental separation [2].

During surgery the foetal is exposed to the effects of anaesthetic agents. Commonly used anaesthetics, including nitrous oxide, enflurane, barbiturates and narcotics, have been extensively used safely in pregnancy. Major operations can be safely performed throughout gestation with a $1-3 \%$ risk of foetal loss and a minimally increased relative risk (1.5-2.0) for low birth weight and premature delivery [7].

Risks to the foetal during surgery are not just anaestheticrelated, but also include intraoperative complications, such as hypoxia and hypotension. Decreased placental perfusion secondary to long-term supine positioning of the mother is a mechanical problem in late pregnancy.

Additionally, postoperative problems, such as fever, infections, gastrointestinal problems and changes in nutritional intake, thrombosis, and pulmonary embolus could have serious adverse effects on foetal well being [8].

If possible, surgery in pregnancy should be scheduled for the second trimester when there is the least risk of foetal harm, or of inducing abortion or premature labour. Involvement of the obstetrician and the neonatologist for foetal monitoring during surgery is invaluable. Treatment to improve foetal lung maturity should be administered where surgery carries a risk of precipitating premature delivery.

\section{Radiotherapy during pregnancy}

The developing human embryo and foetus are extremely sensitive to ionizing radiation, which might cause pregnancy loss, malformations, growth retardation and neurobehavioural defects as described in the earlier section. Radiation doses used in cancer treatment are in the range of 30-70 Gy. However, the effective foetal dose depends on the size of the irradiation field, the target dose, the distance from the foetus to the field, the specific radiation machine, internal scatter, leakage radiation from the tube head, scatter from the collimator, and the shielding used.

In general, radiation therapy should be avoided in a pregnant patient. Patients who have cancers that are remote from the pelvis may be treated with radiation, e.g. breast cancer, supradiaphragmatic Hodgkin's lymphoma, head and neck cancer and brain tumours. Irradiation of the maternal breast or chest wall will expose the foetus to $0.1-0.3 \%$ of the total dose (usually $50 \mathrm{~Gy}$ ). Accordingly, the estimated foetal dose without shielding will be $30 \mathrm{mGy}$ at 8 weeks of gestation, $200 \mathrm{mGy}$ at 24 weeks and $1.4 \mathrm{~Gy}$ at 36 weeks of pregnancy, for treatment with a 6-25 MeV linear accelerator [9].

Cancers in the pelvis cannot be treated adequately with radiation during pregnancy without severe or lethal consequences for the foetus. If possible, radiotherapy should be delayed until the postpartum period. The use of adjuvant and neoadjuvant chemotherapy can delay the initiation of radiotherapy. Advances in neonatology and critical care have made it possible for neonates to survive after being delivered at 32 weeks of gestation. Thus an elective delivery at 32 weeks can be planned and radiotherapy commenced after delivery.

\section{Chemotherapy during pregnancy}

The use of chemotherapy during pregnancy is a topic which raises the maximum controversy. The administration of chemotherapy during pregnancy can induce harmful effects to the foetus. The spectrum of damage includes spontaneous abortion, malformations, mutations, carcinogenesis, organ toxicity and retarded development. The impact of chemotherapy on foetal health depends on the type, duration and dose of drugs given, as well as the gestational period at which the drugs are delivered. The correct dosing of chemotherapy in pregnant women is complicated by the fact that plasma volume, hepatorenal function and albumin concentration change during pregnancy. Most chemotherapy drugs can cross the placenta and enter the foetal circulation. The foetal liver is able to metabolise substrates, and the foetal kidney may be involved in the elimination of drugs. It is possible that a drug excreted into the amniotic fluid may be ingested by the foetus and 
reabsorbed from the gastrointestinal tract. This mechanism could potentiate the effect of a drug exposure on the foetus if the drugs or their breakdown products are excreted in an active form. The placenta is also a route of drug elimination, as it serves as a mechanism to remove waste products and toxins from the foetus. Thus far no pharmacokinetic studies of chemotherapy during pregnancy are available.

During the first 2-4 weeks from conception, toxic injuries result in either no effect or embryonal growth arrest and death (abortion). Later on and during the first trimester of pregnancy, chemotherapy may interfere with organogenesis, with the risk of malformations being maximal $(10 \%$ for single agents, $20 \%$ for combinations) [10,11]. During the second and third trimesters organogenesis is complete with the exception of the CNS and gonads. Consequently, toxic effects seen are functional disorders, intrauterine growth retardation, still birth, premature delivery, low birth weight and maternal or neonatal myelosuppression (incidence 10 $30 \%)$ [12].

Doll et al. [13] have reported that the incidence of foetal malformations with first trimester chemotherapy exposure ranged from $14 \%$ to $19 \%$. Exposure in the second or third trimester was associated with an incidence of foetal malformations of $1.3 \%$. In the general population, the incidence of major congenital malformations has been reported as approximately $3 \%$ of all births.

Anthracyclines are among the most commonly used drugs during pregnancy. In a large study analysing 160 patient pregnancies, the foetal outcome was normal in $73 \%$. Abnormalities included malformations (3\%), foetal death $(9 \%)$, spontaneous abortion (3\%), foetal complications $(8 \%)$ and prematurity $(6 \%)$. Foetal death was often directly consecutive to maternal death $(40 \%)$. Unfavorable foetal outcome was significantly more frequent in leukaemia patients. In patients with solid tumours, the first trimester was significantly associated with more complications. The risk of severe foetal toxicity was increased 30 -fold when the dose of doxorubicin per cycle exceeded $70 \mathrm{mg} / \mathrm{m}^{2}$ [14].

The use of methotrexate during pregnancy is contraindicated, as it induces severe malformations known as the "aminopterin syndrome" [13]. The role of taxanes in chemotherapy is still unclear. The data for taxanes is mainly based on case reports and is not meaningful enough to support its safety.

Available information on possible late effects of foetal chemotherapy exposure is reassuring. A large series of 84 children aged 6-29 and exposed to chemotherapy in utero, found no difference in body growth, neurological maturation, scholar progress and IQ in comparison to matched controls [15]. Data on the development of second tumours, on the reproductive potential and integrity of germ-cells of children, adolescents and young adults exposed to chemotherapy in utero is scanty but does not suggest any adverse effects in comparison to the general population [16].

\section{Other treatments}

In addition to chemotherapy, hormonal therapy and supportive care medications are commonly used for the management of the patient with cancer. Tamoxifen is teratogenic in animals and has been associated with 10 cases of foetal abnormalities among 50 pregnant women exposed to it [17]. Its use should be delayed until delivery.

Molecularly targeted agents are increasingly becoming part of the treatment of different types of cancer. Some examples are traztuzumab (used in breast cancer), rituximab (used in lymphomas) and imatinib (used in leukaemias). There exist a couple of case reports on the use of each in a pregnant patient. The information as such is to scant to make any definite recommendations.

Bisphosphonates cross the placenta and they have been incriminated for bone developmental abnormalities as well as derangements of calcium metabolism in animal experiments and should be avoided during pregnancy.

The anti-emetics ondansentron and metoclopramide have been used during gestation without adverse effects to the foetus and were recently found to be safe in this setting in two international prospective trials $[18,19]$.

Erythropoietin does not cross the placenta and seems to be devoid of teratogenic effects in the reported case series of pregnant women [20].

There is a paucity of data on the use of granulocyte colony stimulating factor (GCSF), though experiments in pregnant rats and few case reports did not show detrimental effects on foetal well being [21]. GCSF may be used for the management of febrile neutropenia in pregnant women with cancer but should not be used for the intensification of chemotherapy regimes.

\section{Obstetric care of the cancer patient}

Prenatal care in women diagnosed with cancer during pregnancy should be performed just like in other pregnant women. To correctly estimate the risk to the foetus caused by the mother's cancer treatment, it is essential to define the gestational age and status of the foetus before the beginning of therapy. Nevertheless, it is recommended to supervise women undergoing chemotherapy during pregnancy closely to intervene early if the foetus is perceived to be at risk.

The time of delivery should be balanced according to the need of cancer treatment and the maturation of the foetus. The mode of delivery should be discussed with the patient according to available medical infrastructure, the patient's previous obstetric history and own preferences. The mode of delivery will depend on the location of the tumour - pelvic tumours and cervical tumours are liable to obstruct labour and Lower segment caesarean s ection (LSCS) may have to be performed. Vaginal delivery is better as it has a lower risk of maternal morbidity compared to caesarean section. 
Delay in further chemotherapy, if required, may be avoided with vaginal delivery.

Delivery should be avoided during the maternal leukocyte nadir period. The delivery should be planned usually 2-3 weeks after the last dose of chemotherapy. Chemotherapy should not be given after 35 weeks gestation because spontaneous delivery can occur before the bone marrow has recovered. The delay of delivery for 3 weeks after chemotherapy also allows for foetal drug excretion via the placenta. Chemotherapy administered shortly before delivery might not have been eliminated from the foetus before delivery, and drugs might therefore persist in the newborn. This is especially true for preterm babies, who have a limited ability to metabolise drugs due to the immaturity of the liver and kidneys.

\section{Vertical transmission}

Vertical transmission of cancer is exceptionally rare. From 1866 to 1999,58 cases of documented maternal malignancy metastatic to the placenta and foetus were reported in the western literature. The tumours most commonly seen coexisting with pregnancy are not those most commonly found involving the placenta and foetus. The most likely way for dissemination is through the haematogenous route. The most common tumour metastasising is malignant melanoma, accounting for $30 \%$ of all pregnancy-associated tumours. The second most frequent malignancies are leukaemia and lymphoma followed by carcinoma of the breast and lung $[1,22]$.

\section{Prognosis and follow-up}

Overall, the general consensus is that women diagnosed with cancer during pregnancy do not have a worse prognosis when matched stage for stage with non-pregnant patients. Patients who have a reasonable chance of cure may desire to have children in the future. The treatment for cancer may jeopardize the chances of future childbearing and the patient must be appropriately guided. The options for preservation for fertility are beyond the scope of this article but should be discussed with the patient and instituted during or before her treatment.

\section{Key points}

- Pregnancy-associated cancer is a rare situation best managed in a multidisciplinary setting

- The maternal cure and foetal well being need to be balanced carefully

- Surgical treatment essentially is the same as in a non-pregnant patient
- Radiation therapy is best avoided till after completion of pregnancy

- Most chemotherapy drugs can be given safely in the second and third trimesters of pregnancy

- The obstetric care in such a setting has to be more vigilant

- The prognosis of pregnancy-associated cancer is no worse than stage for stage matched cancer

- Fertility issues after treatment need to be discussed during treatment planning.

\section{References}

1. Pavlidis NA(2002) Coexistence of pregnancy and malignancy. Oncologist 7:279-287

2. Moran BJ, Yano H, Zahir NA, Farquharson M (2007) Conflicting priorities in surgical intervention for cancer in pregnancy. Lancet Oncol 8:536-544

3. Pentheroudakis G, Pavlidis N (2006) Cancer and pregnancy: Poena magna, not any more. Eur J Cancer 4:2126-2140

4. International Commission on Radiological Protection (2000) Pregnancy and medical irradiation. Ann ICRP 30:1-43

5. Nicklas A, Baker M (2000) Imaging strategies in pregnant cancer patients. Semin Oncol 27:623-632

6. Gentilini O, Cremonesi M, Trifiro G, et al. (2004) Safety of sentinel node biopsy in pregnant patients with breast cancer. Ann Oncol 15:1348-1351

7. Mazze R, Kallen B (1989) Reproductive outcome after anesthesia and operation during pregnancy: A registry study of 5405 cases. Am J Obstet Gynecol 161: 1178-1185

8. Doll DC, Ringenberg QS, Yarbro JW (1988) Management of cancer during pregnancy. Arch Intern Med 148: 2058-2064

9. Van der Giessen PH (1997) Measurement of the peripheral dose for the tangential breast treatment technique with Co-60 gamma radiation and high-energy X-rays. Radiother Oncol 42:257-264

10. Buekers TE, Lallas TA (1998) Chemotherapy in pregnancy. Obs Gyn Clin North Am 25:323-329

11. Cardonick E, Iacobucci A (2004) Use of chemotherapy during human pregnancy. Lancet Oncol 5:283-291

12. Zemlickis D, Lishner M, Degendorfer P, et al. (1992) Foetal outcome after in utero exposure to cancer chemotherapy. Arch Intern Med 152:573-576

13. Doll DC, Ringenberg QS, Yarbro JW (1989) Antineoplastic agents and pregnancy. Semin Oncol 16:337-346

14. Germann N, Goffinet F, Goldwasser F (2004) Anthracyclines during pregnancy: embryo-foetal outcome in 160 patients. Ann Oncol 15:146-150

15. Aviles A, Neri N (2001) Hematological malignancies and pregnancy: a final report of 84 children who received chemotherapy in utero. Clin Lymphoma 2:173-177

16. Brent RL (1999) Utilization of developmental basic science principles in the evaluation of reproductive risks from preand postconception environmental radiation exposures. Teratology 59:182-204 
17. Woo JC, Taechin Y, Hurd TC (2003) Breast cancer in pregnancy. Arch Surg 138:91-98

18. Berkovitch M, Mazzota P, Greenberg R, et al. (2002) Metoclopramide for nausea and vomiting of pregnancy. A prospective multicentre international study. Am J Perinatol 19:311-316

19. Einasson A, Maltepe C, Navioz Y, et al. (2004) The safety of ondansentron for nausea and vomiting of pregnancy: A prospective comparative study. BJOG 111:240-243
20. Scott LL, Ramin SM, Richey M, et al. (1995) Erythropoietin use in pregnancy: two cases and a review of the literature. Am J Perinatol 12:22-24

21. Medlock ES, Kaplan DL, Cecchini M, et al. (1993) Granulocyte-colony stimulating factor crosses the placenta and stimulates foetal rat granulopoiesis. Blood 81:916-922

22. Dildy GA, Moise Jr KJ, Carpenter Jr RJ, et al. (1989) Maternal malignancy metastatic to the products of conception: a review. Obstet Gynecol Surv 44:535-540 